\title{
A!
}

This is an electronic reprint of the original article.

This reprint may differ from the original in pagination and typographic detail.

Zubair, Ahmad; Niroula, John; Chowdhury, Nadim; Zhang, Yuhao; Lemettinen, Jori; Palacios, Tomas

Materials and technology issues for the next generation of power electronic devices

Published in:

2020 Device Research Conference, DRC 2020

DOI:

10.1109/DRC50226.2020.9135183

Published: 01/06/2020

Document Version

Peer reviewed version

Please cite the original version:

Zubair, A., Niroula, J., Chowdhury, N., Zhang, Y., Lemettinen, J., \& Palacios, T. (2020). Materials and technology issues for the next generation of power electronic devices. In 2020 Device Research Conference, DRC 2020 [9135183] (Device Research Conference). IEEE. https://doi.org/10.1109/DRC50226.2020.9135183

This material is protected by copyright and other intellectual property rights, and duplication or sale of all or part of any of the repository collections is not permitted, except that material may be duplicated by you for your research use or educational purposes in electronic or print form. You must obtain permission for any other use. Electronic or print copies may not be offered, whether for sale or otherwise to anyone who is not an authorised user. 


\section{Materials and Technology Issues for the Next Generation of Power Electronic Devices}

Ahmad Zubair ${ }^{1}$, John Niroula ${ }^{1}$, Nadim Chowdhury ${ }^{1}$, Yuhao Zhang ${ }^{2}$, Jori Lemettinen ${ }^{3}$, and Tomás Palacios ${ }^{1, *}$

${ }^{1}$ Microsystem Technology Lab, Massachusetts Institute of Technology, 02139-4307, USA, ${ }^{2}$ Virginia Polytechnic Institute and State University, VA 24061 USA, ${ }^{3}$ Aalto University, 02150 Espoo, Finland

Corresponding author:*tpalacios@mit.edu

By 2030 , about $80 \%$ of all US electricity is expected to flow through power electronics. This will require power electronic devices and circuits with much higher efficiency and smaller form-factor than today's silicon-based systems. III-Nitride semiconductors and other ultra-wide bandgap materials are ideal platforms for the new generation of power electronics thanks to the combination of excellent transport properties and the high critical electric field enabled by their wide bandgap [1]. This talk will discuss recent progress in our group in developing high voltage power transistors and diodes based on wide bandgap materials.

Vertical GaN-based FinFET power transistors have a great potential for $1200 \mathrm{~V}$ applications (Figure 1-2)[2]. The high current density, in combination with minimum parasitics, allow these devices to achieve beyond-state-of-the-art switching performance as summarized in Table 1. In addition, their simple structure and absence of p-type layers is expected to significantly lower their manufacturing cost compared to the traditional vertical transistor architectures. The manufacturing cost can be reduced even further by fabricating these devices on either silicon wafers or engineered substrates, although some key trade-offs between leakage currents and performance need to be carefully studied.

The talk will also discuss the opportunities and challenges brought to power electronic devices by ultra-wide bandgap materials beyond GaN. Specifically, some recent results on AlGaNchannel transistors [3] (Figure 3-4) and vertical diamond devices will be benchmarked with the state-of-the-art devices, and a roadmap for future device development will be presented.

The talk will conclude with a summary of recent work on the heterogeneous integration of power switches, gate drivers and control electronics - a key requirement for reaching the full potential of wide bandgap materials. A new GaN CMOS-driver compatible with standard GaN power devices will be demonstrated (Figure 5-6)[4] as an example system.

Acknowledgements - This research was supported by the ARPA-E SWITCHES and PNDIODES programs, monitored by Dr. Isik Kizilyalli, and by the DARPA DREaM program, monitored by Dr. Young-Kai Chen and Dr. Paul Maki.

REFERENCES: [1] H. Amano et al., J. of Phys. D App. Phys, vol. 51, 163001, 2018. [2] Y. Zhang et al., 2017 IEDM, pp. 9.2.1-9.2.4, 2017. [3] J. Lemettinen, et al., " IEEE Electron Dev. Lett., vol. 40, pp. 1245-1248, 2019. [4] N. Chowdhury, et al., 2019 IEDM, pp. 4.6.1-4.6.4, 2019. 


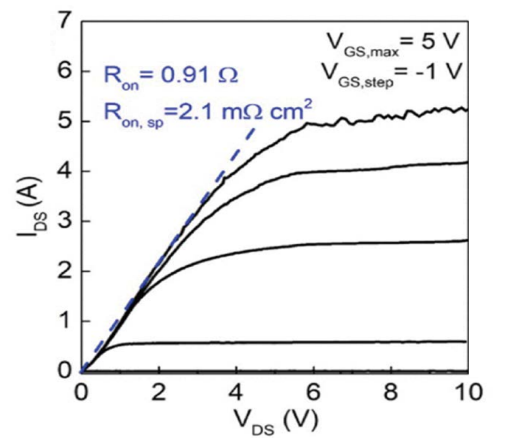

Figure 1: Output characteristics and extracted $R_{o n}$ and $R_{s p, o n}$ in a vertical GaN power FinFET.

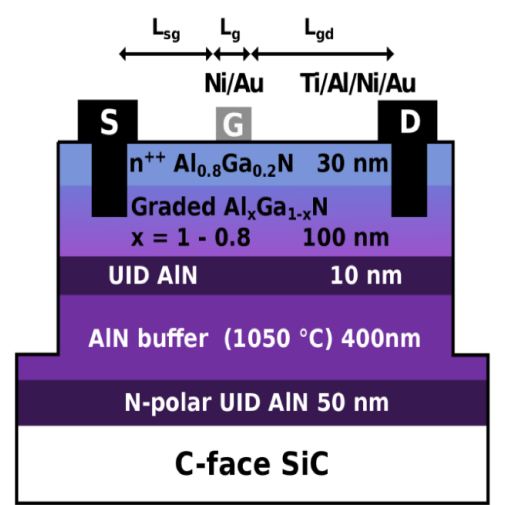

Figure 3: Schematic cross-section of the fabricated N-polar AlGaN/AlN PolFET with recessed contacts.

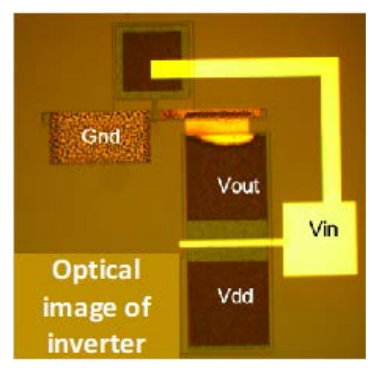

Figure 5: Optical image of a complementary logic GaN inverter fabricated on a GaN-on-Si platform.

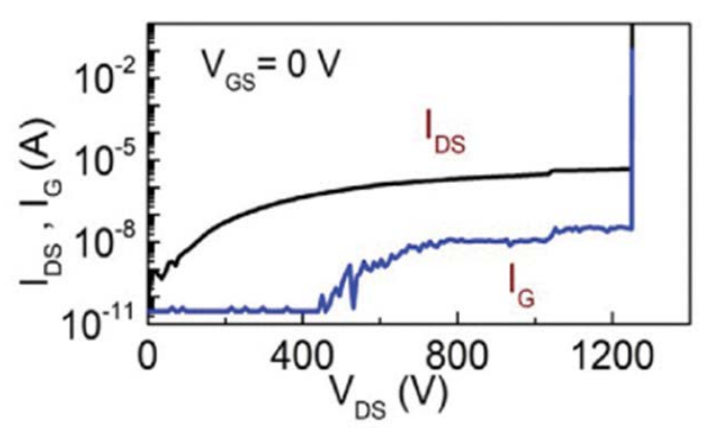

Figure 2: Off-state leakage of a vertical FinFET measured at $V_{\mathrm{GS}}=0 \mathrm{~V}$, demonstrating a breakdown voltage of $1250 \mathrm{~V}$.

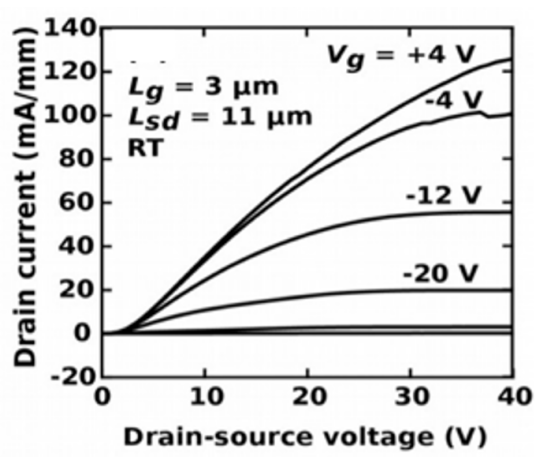

Figure 4: Output characteristics of N-polar PolFET with a 30-nm-thick $\mathrm{Al}_{2} \mathrm{O}_{3}$ gate dielectric.

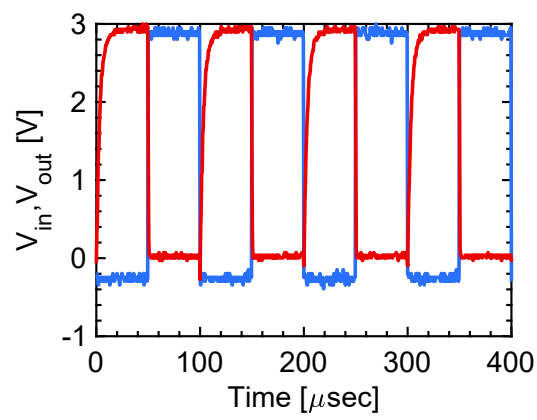

Figure 6: Transient response of the GaN complementary logic inverter shown in Figure 5.

TABLE I. Summary and benchmark of device technologies and their key device metrics for 900-1200 V power switching applications. [2]

\begin{tabular}{|c|c|c|c|c|c|c|c|c|c|c|c|c|}
\hline Device Technology & $\begin{array}{l}\text { Manufacturer/ } \\
\text { Maker }\end{array}$ & $\begin{array}{c}R_{m, m} \\
(\mathrm{~m} \Omega \mathrm{cm}) \\
\left(R_{\mathrm{m}}\right)\end{array}$ & $\begin{array}{l}B V \\
(\mathrm{kV})\end{array}$ & $\begin{array}{l}I_{\text {mat }} \\
(\mathrm{A})\end{array}$ & $\underset{\left(\mathrm{mm}^{2}\right)}{\text { Chip Area }}$ & $V_{\text {т }}(\mathrm{V})$ & $\begin{array}{l}C_{\mathrm{ss}} \\
(\mathrm{pF})\end{array}$ & $C_{\text {oss }}(\mathrm{pF})$ & $\underset{(\mathrm{nC})}{Q_{\bullet}}$ & $\begin{array}{c}Q_{\text {๓⿴囗十 }} \\
(\mathrm{nC})\end{array}$ & $\begin{array}{l}Q_{n_{n}} \\
(\mathrm{nC})\end{array}$ & $\begin{array}{c}\text { Switching FOM }(\mathrm{n} \Omega \cdot \mathrm{C}) \\
R_{m}\left(Q_{\mathrm{c}}+Q_{\omega}\right) \\
R_{m} \cdot\left(Q_{\mathrm{c}}+Q_{\mathrm{m}}+Q_{\mathrm{m}}\right) \\
\end{array}$ \\
\hline $\begin{array}{c}\text { GaN Vertical FinFET } \\
\text { (this work) }\end{array}$ & MIT & $\begin{array}{c}\mathbf{2 . 1} \\
(0.91 \Omega) \\
\end{array}$ & 1.2 & 5 & 0.45 & 1.3 & 248.3 & 42.2 & 1.24 & 2.72 & $\sim 0$ & \begin{tabular}{|c|}
3.3 \\
$\sim 2.0$ (pad optimized) \\
\end{tabular} \\
\hline $\begin{array}{c}\text { SiC MOSFET } \\
(\mathrm{CPM} 2-1200-0160 \mathrm{~B}) \\
\end{array}$ & Cree & $\begin{array}{l}2.7[19] \\
(0.16 \Omega) \\
\end{array}$ & 1.2 & 20 & 6.28 & 2.5 & 525 & 47 & 34 & 14 & 105 & $\begin{array}{c}7.68 \\
24.48 \text { (include } Q_{m} \text { ) } \\
\end{array}$ \\
\hline $\begin{array}{c}\text { SiC JFET Cascode } \\
\text { (UJN1208Z) }\end{array}$ & United SiC & $\begin{array}{c}1.7^{\prime \prime} \\
(0.15 \Omega) \\
\left(5{ }^{2}\right.\end{array}$ & 1.2 & 18.4 & $>1.13^{\prime \prime}$ & 4.4 & 738 & 58 & 30 & 6 & 63 & $\begin{array}{c}5.4 \\
14.85 \text { (include } Q_{\pi} \text { ) }\end{array}$ \\
\hline $\begin{array}{c}\text { Si IGBT } \\
\text { (NGTB15N120FLWG) } \\
\end{array}$ & ON Semi & $\begin{array}{c}\sim 20 \\
(\sim 0.25 \Omega) \\
\end{array}$ & 1.2 & 15 & 320 & 2 & 3600 & 110 & 150 & 68 & 1500 & $\begin{array}{c}54.5 \\
429.5 \text { (include } Q_{m} \text { ) } \\
\end{array}$ \\
\hline $\begin{array}{c}\text { Si CoolMOS } \\
\text { (IPD90R1K2C3) } \\
\end{array}$ & Infineon & $\begin{array}{c}\sim 8^{m-m} \\
(1.2 \Omega) \\
\end{array}$ & 0.9 & 5 & $\sim 23.6$ & 3 & 710 & 35 & 28 & 12 & 3700 & $\begin{array}{c}48 \\
4488 \text { (include } Q_{\varpi} \text { ) } \\
\end{array}$ \\
\hline GaN HEMT Cascode [20] & Transphorm & $(0.19 \Omega)$ & 1.2 & $>20$ & N.A. & 2.3 & N.A. & 43.1 & 10 & N.A. & N.A. & N.A. \\
\hline $\begin{array}{l}\text { Vertical GaN MOSFET [5] } \\
\text { Vertical GaN CAVET [2] }\end{array}$ & $\begin{array}{l}\text { TOYODA GOSEI } \\
\text { Avogy }\end{array}$ & $\begin{array}{l}2.7 \\
2.2 \\
\end{array}$ & $\frac{1.2}{1.5}$ & $\frac{23.2}{2.3}$ & $\frac{2.25}{0.17}$ & $\frac{3.5}{0.5}$ & \multicolumn{6}{|c|}{ N. A. } \\
\hline
\end{tabular}

Note: *@ $V_{\mathrm{w}}=200 \mathrm{~V}$; **Source: http://unitedsic.com/cascodes; ***Calculated by using the $R_{\mathrm{m}}$ in the data sheet and the reported $R_{m, s}$, reflecting the active device area (not including pad/edge areas); ****Source: Infineon's application note titled "CoolMOS ${ }^{\mathrm{w}} \mathrm{C}$ 7: Mastering the Art of Quickness". 